\title{
Aplicação do Teste de Informação, Memória e Concentração (IMC) ao Estudo Epidemiológico de Demência Senil em Fortaleza
}

* Universidade Federal do Ceará, Centro de Ciências de Saúde, Departamento de Farmacologia, Rua Cel. Nunes de Melo, 1.127

Caixa Postal 657

60430 - Fortaleza - CE

* Saúde Comunitária da UFC.

*** Medicina Clínica da UFC.

$* * * *$ Departamento de Estatistica da UFC.
G. S. B. Viana*

M. Z. Rouquayrol**

V. M. S. Bruin $* * *$

J. J. L. Albuquerque ${ }^{* * * *}$

O presente trabalho teve por objetivo verificar a ocorrência de demência senil em amostra de pessoas de 65 e mais anos de idade residentes no Municipio de Fortaleza. Foram testados 865 idosos, a partir do teste de Informação, Memória e Concentração, IMC (teste de Hachinski, modificado), que aborda aspectos concernentes à identificação da pessoa, à memória de fatos atuais e remotos e à concentração, determinando os estados demenciais em função do número e grau de respostas numa seqüencia programada e previamente testada quanto à sensibilidade e especificidade.

Analisando-se os resultados quanto a idade, sexo e condiçâo social, evidenciou-se uma prevalência de $8,4 \%$ de deméncia senil no conjunto geral, com diferença nâo-significativa na faixa de 75 e mais anos de idade (9,3\%), comparada ao grupo de 65 a 74 anos (7,5\%). Os percentuais foram semelhantes para homens e mulheres: $8,7 \%$ e 8,3\%, respectivamente. Quanto à condição social, foi verificado um aumento progressivo nas proporções de estados demenciais, desde $4,2 \%$ para o estrato $A / B$ (abastados), 6,9\% para o grupo $C$ (nivel intermediário) $e$ $10,3 \%$ no nivel $D / E$ correspondente ao estrato de pessoas economicamente desprivilegiadas. Estes resultados, entretanto, deverão ser vistos com certa reserva, dado o grau de recusa por ocasião das entrevistas, especialmente nos estratros $A / B(19,3 \%)$ e $D / E(9,3 \%)$.

Unitermos: Teste de screening, demência senil 


\section{INTRODUÇÃO}

Segundo informe técnico da Organização Mundial da Saúde $^{1}$, o incremento mundial da população de 65 anos e mais, para o período de 1980 até o ano 2000 , será em torno de 138 milhões de pessoas, sendo 100 milhões nas regiões subdesenvolvidas e 38 milhões nas regiões desenvolvidas. A esperança de vida à idade de 60 anos, calculada para o periodo de 1975-78, em países como o Canadá, França ou Suécia, foi da ordem de $22,2 \%$ para mulheres e de $17,5 \%$ para homens com $7,1 \%$ e $3,3 \%$ anos ganhos para pessoas do sexo feminino e masculino respectivamente (considerado o ano de 1990 como base de cálculo dos anos ganhos). $\mathrm{Na}$ América Latina a população idosa terá um acréscimo de 18 milhões em apenas 20 anos, passando de 23 milhões, em 1980 , para 41 milhões no ano 2.000 , correspondente a 6,4 e $7,2 \%$, respectivamente, da população total (Anzola- Perez $^{2}$ ).

No Brasil, projeções do $\mathrm{IBGE}^{3}$ para o período de 1985 2000 estimam que a população de idosos terá um acréscimo de quase 4 milhões, passando de 5,8 para 9,7 milhões num intervalo de apenas 15 anos. Segundo Berquó ${ }^{4}$, a esperança de vida calculada para o Brasil no periodo de 1975-80 à idade de 60-65 anos, foi da ordem de $18,3 \%$ para mulheres e de $17,5 \%$ para homens com $2,6 \%$ e $2,4 \%$ anos ganhos para mulheres e homens, respectivamente (Tabela I). No confronto da população brasileira por grupos etários, evidencia-se decréscimo da população de crianças e adolescentes e aumento progressivo dos grupos de adultos e idosos nos anos de 1970, 1980 e 1990 (Figura I).

Ramos e colaboradores ${ }^{5}$, em trabalho recente sobre envelhecimento populacional destacam a evolução da expectativa de vida para a população de São Paulo, demonstrando que, naquele Município, a esperança de vida ao nascer, que em 1950 era de $57,5 \%$, passou para $67,4 \%$ em 1980 e referem que aqueles que têm 60 anos hoje deveräo viver por mais um periodo de 15 a 20 anos à semelhança do que ocorre em qualquer pais desenvolvido.

No Município de Fortaleza a esperança de vida ao nascer referente ao sexo masculino ascendeu de $50,9 \%$ no período 1966-68 para 59,3\% no período $1978-90$. Aumento idêntico é observado para o sexo feminino cuja esperança de vida passou de 55,1\% para $65,9 \%$ em apenas 12 anos $\left(\right.$ Silva $^{6,7}$ ). 
PESQUISA

\section{TABELA I}

Esperança de Vída, por Sexo, para a População Idosa:

Estimativas por Grupos Etários. Brasil, 1950 - 1980

\begin{tabular}{lrcc}
\hline Sexo e Idade & $1950-55$ & $1975-80$ & $\begin{array}{c}\text { Anos Ganhos de } \\
1950 \text { a } 1980\end{array}$ \\
\hline Homens & 15,10 & 17,53 & \\
$\AA$ idade $60-65$ & 9,54 & 11,27 & 2,43 \\
$\AA$ idade $70-75$ & 5,36 & 6,32 & 1,73 \\
$\AA$ idade $80-85$ & & & 0,96 \\
& & & \\
Mulheres & 15,72 & 18,34 & 2,62 \\
$\AA$ idade $60-65$ & 9,90 & 11,78 & 1,88 \\
$\AA$ idade $70-75$ & 5,53 & 6,61 & 1,08 \\
$\AA$ idade $80-85$ & & & \\
\hline
\end{tabular}

In: Berquo, E. S. \& Leite, V. M. (4)

\section{FIGURA I}

Percentual de Crescimento Demográfico em Diferentes

Faixas Etárias no Brasil

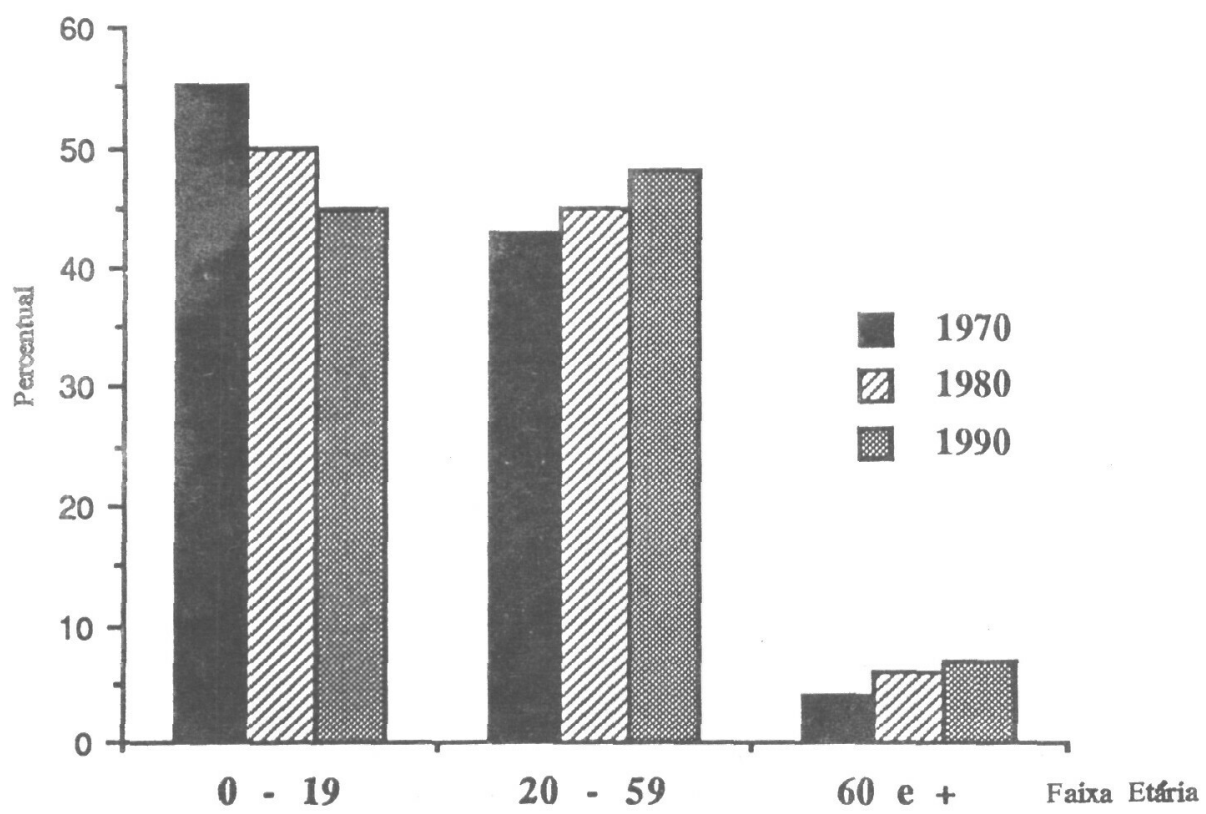

Fonte: IBGE, Anuário de 1989, pp 72/73.

Cadernos de Saúde Pública, RJ, 7(3): 396-408, jul/set, 1991 
Na Tabela II, dos estudos de Silva, observa-se decréscimo das principais taxas de mortalidade por conta de melhoria do nível de saúde em Fortaleza, com conseqüente mudança na estrutura de mortalidade. $\mathrm{Na}$ análise dos dados de óbitos para Fortaleza em diferentes periodos, diz, textualmente, aquele autor: As doenças crônico-degenerativas foram as que experimentaram os maiores incrementos, e apresenta coeficientes padronizados para neoplasias, doenças cérebro-vasculares e outras.

\section{TABELA II}

Indicadores de Saúde no Município de Fortaleza, nos

Períodos 1966-68, 1969-71, 1972-74 e 1978-80

\begin{tabular}{|c|c|c|c|c|c|}
\hline Indicadores & Sexo & $1966-68$ & $1969-71$ & $1972-74$ & $1978-80$ \\
\hline $\begin{array}{l}\text { 1. Mortalidade geral } \\
\text { (por } 1.000 \text { habi- } \\
\text { tantes) }\end{array}$ & $\mathrm{T}$ & 12,72 & 12,15 & 10,97 & 8,00 \\
\hline 2. fndice de Swaroop- & M & 20,15 & 21,97 & 25,44 & 28,77 \\
\hline Uemura (\%) & $\mathrm{F}$ & 22,91 & 25,39 & 29,23 & 34,43 \\
\hline $\begin{array}{l}\text { 3. Mortalidade pro- } \\
\text { porcional em meno- } \\
\text { res de um ano (\%) }\end{array}$ & M & $\begin{array}{l}48,83 \\
44,74\end{array}$ & $\begin{array}{l}48,26 \\
45,25\end{array}$ & $\begin{array}{l}44,17 \\
42,54\end{array}$ & $\begin{array}{l}43,25 \\
43,02\end{array}$ \\
\hline 4. Indice de Guedes & $\begin{array}{l}\mathrm{M} \\
\mathrm{F}\end{array}$ & $\begin{array}{l}-16,41 \\
-13,60\end{array}$ & $\begin{array}{l}-15,09 \\
-12,00\end{array}$ & $\begin{array}{r}-11,95 \\
-8,72\end{array}$ & $\begin{array}{l}-9,85 \\
-5,05\end{array}$ \\
\hline $\begin{array}{l}\text { 5. Mortalidade pre } \\
\text { escolar (por } \\
1.000 \text { habitantes) }\end{array}$ & $\begin{array}{l}\mathrm{M} \\
\mathrm{F}\end{array}$ & $\begin{array}{l}17,12 \\
18,22\end{array}$ & $\begin{array}{l}13,55 \\
14,35\end{array}$ & $\begin{array}{r}9,86 \\
10,56\end{array}$ & $\begin{array}{l}4,43 \\
4,79\end{array}$ \\
\hline $\begin{array}{l}\text { 6. Mortalidade infan- } \\
\text { til (por } 1.000 \\
\text { nascidos vivos) }\end{array}$ & $\begin{array}{l}\mathrm{M} \\
\mathrm{F}\end{array}$ & $\begin{array}{l}170,13 \\
145,09\end{array}$ & $\begin{array}{l}162,73 \\
137,91\end{array}$ & $\begin{array}{l}139,31 \\
117,49\end{array}$ & $\begin{array}{r}116,00 \\
97,21\end{array}$ \\
\hline $\begin{array}{l}\text { 7. Mortalidade mater } \\
\text { na (por } 10.000 \\
\text { nascidos vivos) }\end{array}$ & & 8,11 & 7,84 & 6,66 & 7,88 \\
\hline $\begin{array}{l}\text { 8. Esperança de vida } \\
\text { ao nascer (em anos) }\end{array}$ & $\begin{array}{l}M \\
F\end{array}$ & $\begin{array}{l}50,93 \\
55,12\end{array}$ & $\begin{array}{l}51,38 \\
56,42\end{array}$ & $\begin{array}{l}53,38 \\
58,79\end{array}$ & $\begin{array}{l}59,31 \\
65,93\end{array}$ \\
\hline
\end{tabular}

FONTE: Silva, M. G. C. $(6,7)$.

O presente estudo, ao aplicar o teste de Informação, Memória e Concentração (IMC) em amostra de idosos do Cadernos de Saúde Pública, RJ, 7(3): 396-408, jul/set, 1991 
Municipio de Fortaleza, teve por objetivo conhecer a prevalência de demência senil e sua distribuição quanto a idade, sexo e estrato social. O IMC foi adaptado de Hachinski et al. ${ }^{8}$ e testado em trabalho anterior (Viana et al. ${ }^{9}$ ) quanto à especificidade e sensibilidade, com resultados indicando ser este teste um adequado instrumento na deteç̧ão de estados demenciais em idosos.

\section{METODOLOGIA}

O estudo foi realizado em Fortaleza, no periodo de novembro-88 a fevereiro-89. O município apresenta uma população total estimada para 1988 de 1.736 .103 , sendo cerca de 81.500 habitantes com idade igual ou superior a 65 anos. Foram entrevistadas, com respectiva aplicação do teste IMC, 865 pessoas nessa faixa etária, sendo $36,1 \%$ do sexo masculino e $63,9 \%$ do sexo feminino, adotando-se para a classificação dos estratos sociais os critérios da Associação Brasileira de Pesquisa de Mercado (Abipeme), com classificação A, B, C, D e E para as diversas categorias, de acordo com o nivel de instrução, a posse e o uso de bens e serviços (carro, empregada doméstica etc.) e tipo de habitação. Por serem semelhantes, os grupos A e B bem como $D$ e $E$ foram fundidos em $A / B$ e $D / E$.

A população idosa foi entrevistada a nível domiciliar por uma equipe de estudantes universitários previamente treinados, e a entrevista consistiu na aplicação do teste de Informação, Memória e Concentração (IMC), de acordo com Hachinski ${ }^{8}$, modificado por Viana e colaboradores ${ }^{9}$, e já testado anteriormente em idosos do Lar Torres de Melo, em Fortaleza. O IMC modificado é composto de 37 questōes, adaptadas às condições locais, apresentando 14 itens sobre informações pessoais e ambientais, 8 itens sobre memória remota, 9 sobre memória recente e 6 referentes à concentração. Escores com diferentes pesos são atribuídos às questões, perfazendo um total de 37 quando todas as perguntas são respondidas satisfatoriamente. Para fins de computação, a linha divisória foi estabelecida arbitrariamente numa regiāo intermediária do teste (linha 19), a partir da escala total de 37 . Assim, os que atingiram valores de 20 ou mais foram considerados como não-portadores de estados demenciais. A sensibilidade de $86,1 \%$ e a especificidade de $82,9 \%$ foram consideradas satisfatórias, segundo Viana et al. ${ }^{9}$ no referido trabalho. 
Quanto à amostragem, foram feitos sorteios dos bairros considerados, grosso modo, do tipo A/B (onde moram pessoas abastadas) como, por exemplo, o bairro chamado Aldeota; tipo C (intermediário), como é o caso da Cidade 2000 e tipo D/E, onde estão incluídas as favelas e conjuntos periféricos. Além deste critério, após amostragem aleatória das ruas e, em cada rua, amostra sistemática das casas, foram levados em consideração o tipo de habitação e a posse e o uso de bens e serviços, já referidos anteriormente. Antes, entretanto, de fazer anotações na ficha individual, explicava-se o objetivo do estudo e perguntava-se, em cada domicílio, se havia idosos com 65 anos ou mais de idade e sobre a possibilidade de entrevista com preenchimento, pelo entrevistador, do questionário (teste IMC).

Para obtenção de amostra de pelo menos $1 \%$ dos idosos de Fortaleza, foram visitadas 3.676 residências e entrevistadas 1.097 pessoas com 65 ou mais anos de idade (em cerca de $70 \%$ dos domicílios não havia indivíduos nessa faixa etária). A taxa de aceitação à entrevista com respostas especificas ao questionário foi de $78,9 \%$. As causas de não-participação de alguns idosos (232) referem-se principalmente a: recusa $(9,3 \%)$, ausência $(5,0 \%)$ e outros motivos $(6,8 \%)$ tais como doença, hospitalização ou porque estavam dormindo (Tabela III). Não houve revisita.

A partir das taxas especificas de demência por estrato, idade e sexo, foi utilizado o teste estatístico de proporções (ao nível de 5\%), para fins de análise e comparação dos dados.

\section{TABELA III}

Percentuais de Respostas das Pessoas Idosas ao Teste

IMC por Ocasiăo das Visitas Domiciliares, segundo

os Estratos A/B (Abastado), C (Intermediário) e D/E (Pobre) - Fortaleza, 1988

\begin{tabular}{|c|c|c|c|c|c|c|c|c|}
\hline \multirow{3}{*}{$\begin{array}{l}\text { Respostas } \\
\text { ao Teste }\end{array}$} & \multicolumn{6}{|c|}{ Estratos } & & \\
\hline & \multicolumn{2}{|c|}{$A / B$} & \multicolumn{2}{|c|}{$\mathrm{C}$} & \multicolumn{2}{|c|}{$D / E$} & \multicolumn{2}{|c|}{ Total } \\
\hline & $\mathrm{n}:$ & $\%$ & $\mathbf{n}:$ & $\%$ & n: & $\%$ & n: & $\%$ \\
\hline Responderam & 120 & 57,9 & 261 & 80,5 & 484 & 85,5 & 865 & 78,9 \\
\hline Recusaram & 40 & 19,3 & 10 & 3,1 & 52 & 9,2 & 102 & 9,3 \\
\hline Ausentes & 15 & 7,0 & 33 & 10,2 & 8 & 1,4 & 55 & 5,0 \\
\hline Outros motivos & 33 & 15,8 & 20 & 6,2 & 22 & 3,9 & 75 & 6,8 \\
\hline Total & 207 & 100,0 & 324 & 100,0 & 566 & 100,0 & 1.097 & 100,0 \\
\hline
\end{tabular}




\section{RESULTADOS}

Considerando o teste IMC como válido na deteç̧ão de demência senil (Viana e colaboradores ${ }^{9}$ ) e julgando ser a amostra de idosos representativa da população do Município de Fortaleza, foi evidenciada uma taxa de prevalência de demência igual a $8,4 \%$, entre pessoas de 65 e mais anos de idade. Comparando-se os dois grupos formados pelos menos idosos (65 a 74 anos) e os mais idosos (75 e +), o teste pôs em evidência uma leve diferença (estatisticamente não-significante), com taxa de demência de $9,3 \%$ para o grupo mais idoso e de $7,5 \%$ para o menos idoso (Tabela IV). Não houve diferença na frequiência de demência entre homens e mulheres, sendo as proporçōes semelhantes, $8,7 \%$ para o sexo masculino e 8,3\% para of feminino (Tabela V).

\section{TABELA IV}

Proporção de Demência em Amostra de Idosos de Acordo com dois Grupos Etários. Município de Fortaleza, 1988

\begin{tabular}{lllllll}
\hline Grupo Etário & \multicolumn{2}{c}{ Deméncia } & \multicolumn{2}{c}{ Não-Deméncia } & \multicolumn{2}{c}{ Total } \\
& $\mathrm{n}$ & $\%$ & $\mathrm{n}$ & $\%$ & $\mathrm{n}$ & $\%$ \\
\hline & & & & & & \\
$65-74$ & 30 & 7,5 & 372 & 92,5 & 402 & 100,0 \\
75 e mais & 43 & 9,3 & 420 & 91,5 & 463 & 100,0 \\
\hline Total & 73 & 8,3 & 792 & 91,6 & 865 & 100,0 \\
\hline
\end{tabular}

\section{TABELA V}

Proporção de Demência, segundo Sexo, em Amostra da População Idosa do Município de Fortaleza, 1988

\begin{tabular}{lcccccc}
\hline \multirow{2}{*}{ Sexo } & \multicolumn{2}{c}{ Demência } & \multicolumn{2}{c}{ Não-Demência } & \multicolumn{2}{c}{ Total } \\
& $\mathrm{n}$ & $\%$ & $\mathrm{n}$ & $\%$ & $\mathrm{n}$ & $\%$ \\
\hline & & & & & & \\
Masculino & 27 & 8,7 & 285 & 91,3 & 312 & 100,0 \\
Feminino & 46 & 8,3 & 507 & 91,7 & 553 & 100,0 \\
\hline Total & 73 & 8,4 & 792 & 91,6 & 865 & 100,0 \\
\hline
\end{tabular}


Quanto ao estrato social, considerando-se as categorias $\mathrm{A} / \mathrm{B}$ (abastada), C (intermediária) e $\mathrm{D} / \mathrm{E}$ (pobre), verificou-se um aumento crescente de demência em direção aos estratos sociais mais pobres. Assim a proporção de demência no estrato A/B foi de $4,2 \%$, aumentando esse resultado para $6,9 \%$ no estrato $\mathrm{C}$ e ampliando ainda mais para o estrato $\mathrm{D} / \mathrm{E}$, formado por pessoas das camadas periféricas desprivilegiadas, que apresentaram taxa de 10,3\% (Tabela VI), diferença significante quando comparados os estratos $\mathrm{A} / \mathrm{B}$ e $\mathrm{D} / \mathrm{E}$, independente de sexo e idade.

\section{TABELA VI}

Proporçāo $\left({ }^{*}\right)$ de Demência, segundo Estratos, em Amostra de Idosos do Município de Fortaleza, 1988

\begin{tabular}{ccccccc}
\hline & \multicolumn{2}{c}{ Demência } & \multicolumn{2}{c}{ Não-Demência } & \multicolumn{2}{c}{ Total } \\
Estrato & $\mathrm{n}$ & $\%$ & $\mathrm{n}$ & $\%$ & $\mathrm{n}$ & $\%$ \\
\hline $\begin{array}{c}\mathrm{A} / \mathrm{B} \\
(\text { Abastado }) \\
\mathrm{C}\end{array}$ & 5 & $4,2(\mathrm{a})$ & 115 & 95,8 & 120 & 100,0 \\
$\begin{array}{c}\text { (Intermediśrio) } \\
\text { D/E } \\
(\text { Pobre })\end{array}$ & 18 & $6,9(\mathrm{a}, \mathrm{b})$ & 243 & 93,1 & 261 & 100,0 \\
\hline
\end{tabular}

(*) Duas proporçōes seguidas pela mesma letra não diferem estatisticamente entre si, ao nfvel de $5 \%$ de probabilidade (teste $\mathrm{Z}$ ).

Obs.:Os intervalos de confiança para a proporção de demencia foram calculados em cada estrato com os seguintes valores: A/B $[0,6 \cdot 7,5] ; C[3,8 \cdot 10,0]$ e D/E $[7,6 \cdot 13,1]$.

A Tabela VII oferece uma visão de conjunto de toda a amostra, num total de 865 idosos testados frente ao IMC, sendo 553 mulheres e 312 homens. O maior número de mulheres, tanto na amostra quanto no universo, é indicativo da maior sobrevida da população feminina em idades avançadas, em relação à população masculina. A distribuição segundo o estrato foi de 120 pessoas na classe $A / B$ (abastada), 261 na $C$ (intermediária) e 484 na D/E (pobre). Essa amostra foi considerada equivalente ao recomendado pelo informe da $\mathrm{OMS}^{1}$, que fixa os grupos amostrais em torno de $15 \%$ para a classe abastada, $35 \%$ para a média e $55 \%$ para a pobre.

Cadernos de Saúde Pública, RJ, 7(3): 396-408, jul/set, 1991 


\section{PESQUISA}

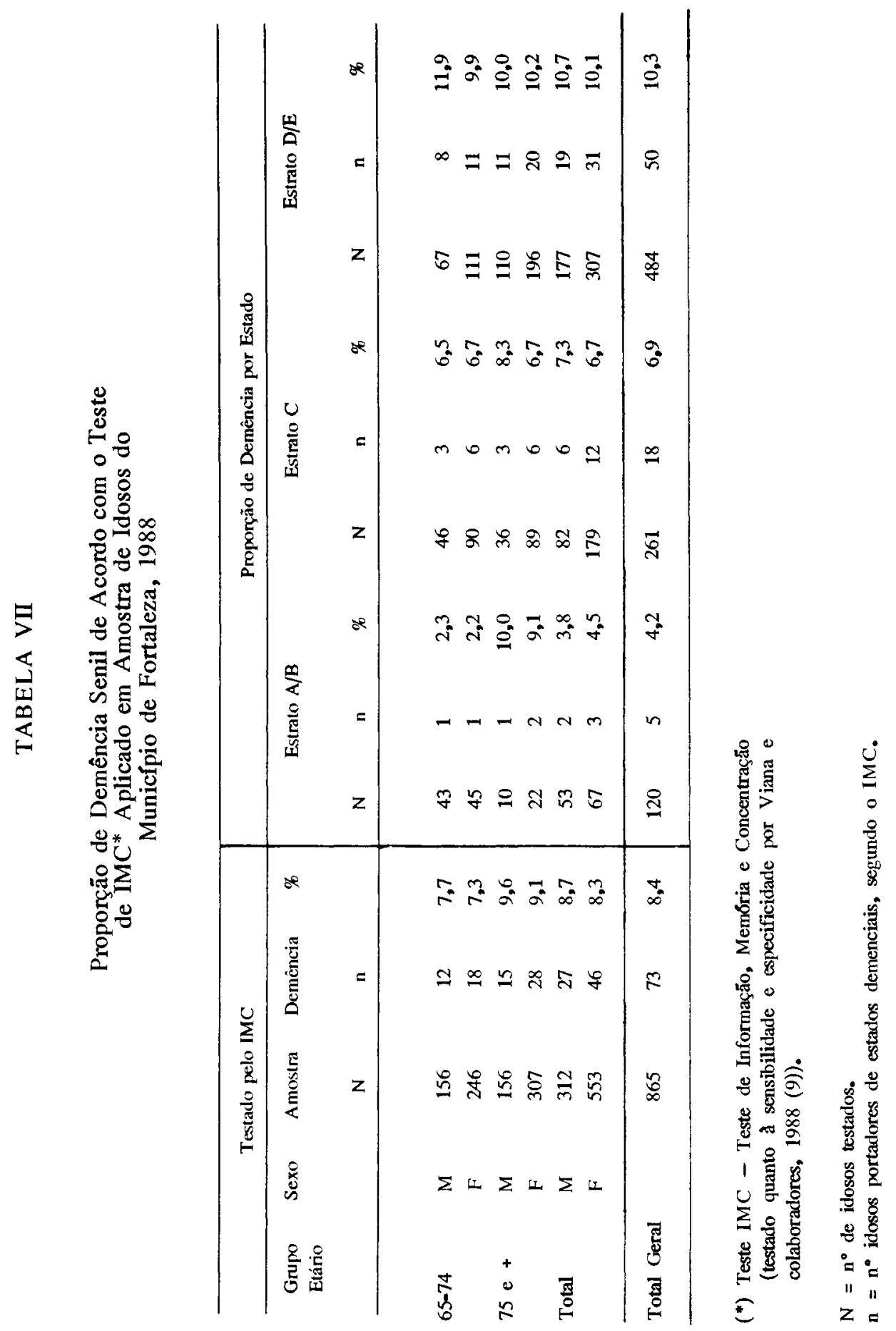




\section{DISCUSSÃO}

A demência senil, que acomete com maior freqüência pessoas com 65 e mais anos de idade, é doença crônica, lenta e progressiva, com degradação das funções mentais superiores, incluindo, entre outros aspectos, perda da memória recente e da capacidade de interpretar ou mesmo de repetir frases completas, dificuldade para resolver problemas do cotidiano, comprometimento da linguagem e da comunicação e desorientação no tempo e no espaço ${ }^{10,11}$.

Dentre os transtornos demenciais da velhice, a Classificação Internacional de Doenças, na sua $9^{2}$ revisão ${ }^{12}$, inclui no item 290, sob a rubrica "Psicoses Orgânicas Senis e Présenis", categorias independentes desde as mais simples até o grupo sem definição precisa $(290,9)$, sendo as mais comuns: demência senil simples $(290,0)$, demência pré-senil $(290,1)$, demência do tipo depressivo $(290,2)$ ou do tipo confusional $(290,3)$ e demência arteriosclerótica $(290,4)$.

Em estudo epidemiológico realizado por Almeida Filho e colaboradores ${ }^{13}$ em Salvador-Bahia, observou-se prevalência de $6,8 \%$ no tocante aos casos diagnosticados como "quadros orgânico-cerebrais" em amostra de 44 pessoas com mais de 65 anos de idade. Esta cifra encontrada em idosos é 14 vezes maior do que no restante da população amostrada, revelando ser este grupo muito mais vulnerável a patologias associadas a degeneraçōes morfológicas e funcionais relacionadas à própria senectude.

No presente estudo o grupo mais idoso ( $75 \mathrm{e}+$ anos) apresentou proporção maior de demência $(9,3 \%)$, em comparação com os menos idosos (65-74 anos) cuja taxa foi de 7,5\%. Segundo o Royal College of Physicians ${ }^{14}, 5$ a $7 \%$ das pessoas com mais de 65 anos têm alguma forma de demência, sem fazer referência à classe social. No presente trabalho verificou-se aumento progressivo das taxas de demência de acordo com as condições sócio-econômicas das famílias, tendo sido observada uma menor proporção de casos entre pessoas abastadas $(4,2 \%)$ e maior proporção $(10,3 \%)$ entre aquelas pertencentes às classes desprivilegiadas. Do mesmo modo uma maior proporção de demência $(10 \%)$ foi observada no grupo de idosos analfabetos ou semi-alfabetizados, enquanto que apenas $2 \%$ de demência foi detectada no grupo de idosos pertencentes ao nível educacional acima do primário. Vale ressaltar que o nível educacional é um dos critérios embutidos na determinação do estrato social.

Cadernos de Saúde Pública, RJ, 7(3): 396-408, jul/set, 1991 
Berquó $^{4}$ registra alto indice de analfabetismo entre os idosos. No que tange a esse aspecto, Sampaio e Colaboradores $^{15}$, em estudo sobre migração e doença mental, informam a existência de $88 \%$ de analfabetos entre os migrantes que se instalaram na periferia de Fortaleza e que, posteriormente, foram atendidos no ambulatório de saúde mental da cidade.

Comparando-se a proporção de demência no presente estudo $(8,4 \%)$ com o percentual encontrado anteriormente (Viana et al. ${ }^{9}$ ) entre idosos residentes em asilo de Fortaleza $(52 \%)$, verifica-se que no asilo há uma concentração de casos, por se tratar de uma instituição para atendimento à velhice desamparada. Segundo Erkinjunki ${ }^{16}$, a ocorrência de demência em indivíduos confinados excede àquela da população em geral, podendo ser superior a $30 \% \mathrm{em}$ indivíduos com idade igual ou superior a 85 anos.

Segundo informe 665 da Organização Mundial de Saúde, Problemas de moradia, nutrição deficiente e outros poderão fazer com que as pessoas dessas classes estejam mais sujeitas a problemas psico-orgánicos na velhice ${ }^{13}$. Desta afirmativa depreende-se que o contexto social, além do fator idade, poderá influenciar nas taxas de demência.

Há previsōes de que, até o final deste século, dois terços da população de idosos do mundo estejam em países subdesenvolvidos, sendo mister o empreendimento de programas de intervenção apropriados, com vistas a se tentar conseguir uma relativa autonomia do idoso e o prolongamento de sua vida útil.

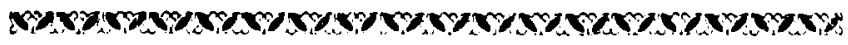

The objective of the present paper was to verify the occurrence of senile dementia in the city of Fortaleza, Brazil. A screening test, the Information, Memory and Concentration Test (IMC), was applied to 865 persons ( 65 years old and older). The Test includes questions on personal identification, recent and remote memory and concentration. Dementia was determined as a function of the total score obtained by the person in the test previously examined as far as sensitivity and specificity are concerned. The results were analysed according to age, sex and social strata. It was demonstrated a prevalence of dementia of $8.4 \%$ in the sample studied 
with no significant difference in the proportions of cases among people 75 years old or older (9.3\%) as compared to the group younger, 65 to 74 years old, (7.5\%). The percentage of dementia was similar between men (8.7\%) and women $(8.3 \%)$. It was verified a progressive increase in the frequence of dementia ranging from $5.2 \%$ for the higher social classes $(A / B)$ to $6.9 \%$ and $10.3 \%$ for medium $(C)$ and lower $(D / E)$ ones respectively, with a significant difference between the social classes $A / B$ and $D / E$. These results, however, should be seen with caution because of the percentage of refuse especially in the higher, $A / B(19.3 \%)$ and lower $D / E(9.3 \%)$ social classes.

\section{REFERÊNCIAS BIBLIOGRÁFICAS}

1 - ORGANIZAÇÃO MUNDIAL DE SAÚDE - Aplicaciones de la epidemiología al estudio de los ancianos. Série de Informes Técnicos, 706, Genebra, 1984.

2 - ANZOLA-PEREZ, E. El envejecimiento en América Latina y el Caribe. OPS, Publ. Cient. 492, 1985.

3 - IBGE - Anuário Estatístico do Brasil, Rio de Janeiro, 1989.

4 - BERQUÓ, E. S. \& LEITE, V. M. - Algumas considerações sobre a demografia de população idosa no Brasil. Ciência e Cultura, 40 (7): 679-688, 1988.

5 - RAMOS, L. R.; VERAS, R. P. \& KALACHE, A. - Envelhecimento populacional: uma realidade brasileira. Rev. Saúde Pública. São Paulo, 21 (3): 211-224, 1987.

6 - SILVA, M. G. C. - Situação de Saúde em Fortaleza, Análise através da mortalidade 1978-80. IOCE, Fortaleza, 1982. 205p.

7 - _ - Situação de Saúde em Fortaleza. Análise através da mortalidade em 1966-74. IOCE, 1990. 177p.

8 - HACHINSKI, V. C.; McALLISTER, V. L. \& RUSSEL, R. W. R. Cerebral blood flow in dementia. Arch. Neurol. 32: 632-637, 1975.

9 - VIANA, G. S. B.; BRUIN, V. M. S.; LEITÃO, M. C. A. \& ROUQUAYROL, M. Z. Adaptação de um teste de "screening" para a detecção de demência. J. Bras. Psiq. 37(1): 29-31, 1988.

10 - ORGANIZAÇÃO MUNDIAL DE SAÚDE - La demencia en la edad avanzada: investigación y acción. Série de Informes Técnicos. Genebra, 730, 1986.

11 - _ - El envejecimiento neuronal y sus relaciones con la patología neurológica humana. Genebra, Série de Informes Técnicos 665, 1981.

Cadernos de Saúde Pública, RJ, 7(3): 396-408, jul/set, 1991 
12 - - Classificação Internacional de Doenças. 92 Revisão (1975) Centro da OMS para classificação de doenças em Português, São Paulo, 1985. 809p.

13 - ALMEIDA FILHO, N; SANTANA, V. S. \& PINHO, A. R. Estudo epidemiológico dos transtornos mentais em uma população de idosos. Área urbana de Salvador-Bahia. J. Bras. Psiq. 33(2): 114-120, 1984.

14 - College commitee on geriatrics: organic mental impairment in the elderly. J. Royal College of Phys. London, 15 (3): 141-167, 1981.

15 - SAMPAIO, J. J. C.; CARDOSO, M. H. P. \& CORDEIRO, M. L. M. B. Migração e doença mental (Revisão da bibliografia cearense, sobre o tema até 1980). Revista Cearense de Psiquiatria, Ano III - n. 3, 35-50, 1981.

16 - ERKINJUNKI, T.; WIKSTRON, J.; PAULO, J.; AUTIO, L. Dementia among medical inpatients. Evaluation of 2000 consecutive admissions. Arch. Intern. Med. 146, 19231926, 1986. 